\title{
Effect of Synthesis Time of Carbon Supported Pd/Au NPs on TCE degradation
}

\author{
Candice Stauffer ${ }^{1}$, Kavita Meduri², Graham O'Brien Johnson ${ }^{3}$, Paul G. Tratnyek ${ }^{3}$ and Jun Jiao ${ }^{1,2}$.
}

1. Department of Physics, Portland State University, Portland, OR, USA.

2. Department of Mechanical \& Materials Engineering, Portland State University, Portland, OR, USA.

3. Institute of Environmental Health, Oregon Health \& Science University, Portland, OR, USA.

The synthesis of palladium $(\mathrm{Pd})$ and gold $(\mathrm{Au})$ nanoparticles $(\mathrm{NPs})$ for the treatment of groundwater has become a popular method because of its effectiveness and sustainability [1]. An effective synthesis method for the production of these NPs uses a combination of sonication and solvothermal processes [3], [4]. The as-made samples are designed to have a 2:1 Pd:Au molar ratio, and a $5 \mathrm{wt} \%$ of $\mathrm{Pd}$ on carbon support, for comparison with corresponding commercial granular activated carbon (GAC) supported $5 \mathrm{wt} \% \mathrm{Pd}$ catalysts. In the past, we have shown that these as-made GAC supported Pd/Au NPs which have been fabricated in $24 \mathrm{hr}$ were potent at degrading carcinogens like trichloroethylene (TCE) [3],[4]. Here, we have investigated the effect of formation time on carbon-supported $\mathrm{Pd} / \mathrm{Au} \mathrm{NPs}$ catalyst to potentially expedite the synthesis process. We synthesized samples in one-hour intervals between 0 and $24 \mathrm{hr}$ and here compare the 1-hr and 24-hr samples. The samples were characterized using an FEI Tecnai F-20 TEM/STEM with EDS capabilities and a Shimadzu UV 3600 UV-Vis Spectrophotometer. Specifically, for TEM analysis, for better characterization, graphene was used as a support in the place of the denser, thicker GAC. In doing so, the surface of GAC is approximated to be similar to a highly defective surface of graphene. For any TCE degradation testing however, the as-made GAC-supported Pd/Au NPs have been used.

The images in Figure 1 and Figure 2 show Pd and Au NPs supported by graphene. Figure 1 of a sample that was fabricated in $24 \mathrm{hr}$, shows a cluster of Pd NPs surrounding an Au core. This structure is confirmed by STEM dark-field (Figure 3). The structure is consistently found throughout the surface of graphene support. These NPs resemble the expected structure of Pd/Au NPs [2],[3]. Likewise, after only $1 \mathrm{hr}$ of synthesis (Figure 2), these similar clusters were observed (Figure 2b). In addition, the sample also contains what appears to be "less developed" NPs (Figure 2c) which have not grown to the size of the larger NPs in Figure 2b. This $\mathrm{Pd}_{\text {shell }} \mathrm{Au}$ core structure appears to be the same; however, the Pd coating is attenuated. For further characterization, both samples were synthesized without support and isolated in DI-water for UV-Vis analysis. A higher absorption for Pd/Au NPs were found in the 24-hr sample compared with the 1-hr sample (Figure 4), observed at the expected peak around 500-600nm [5]. This suggests that despite an incomplete formation of the cluster at $1 \mathrm{hr}$, the catalyst still contains $\mathrm{Pd} / \mathrm{Au}$ NPs to degrade TCE. The1hr sample differs from the 24-hr sample in that some of the NPs on their carbon support do not fully form in only $1 \mathrm{hr}$. With an additional $23 \mathrm{hrs}$, the NP clusters grow in size and as a result loses surface area that is able to react with the any surrounding contaminate. This could result in a 1-hr sample potentially be capable of degrading TCE and other contaminants even more efficiently than the 24-hr sample. Since the Pd loading is kept consistent with that of commercial catalysts, the addition of Au used in this synthesis process appears to produce a more potent catalyst. In conclusion, when Au is used as a promoter, lowering the Pd loading does not compromise the catalytic activity and performance of the catalyst even though a shorter time of synthesis causes the NPs to be smaller. That is, while synthesis time affects the size of the $\mathrm{Pd} / \mathrm{Au}$ NPs cluster, it does not affect the performance of the catalyst. To further examine the performance of the catalyst, a systematic analysis of TCE degradation using GAC-supported catalysts made over different synthesis times is currently being tested using gas chromatography. Preliminary results are in agreement with the results presented here. 
References:

[1] BP Chaplin, M Reinhard, WF Schneider, C Schu, JR Shapley, TJ Strathmann, and CJ Werth, Environ. Sci. Technol. 46 (2012), p. 3655.

[2] Y Mizukoshi, K Okitsu, Y Maeda, TA Yamamoto, R Oshima, and Y Nagata, J. Phys. Chem. B 101 (1997), p. 7033.

[3] K Meduri et al, Microsc. Microanal. (2017), p. 1916.

[4] K Meduri et al, Microsc. Microanal. (2016), p. 332.

[5] Zhu, L. D., Zhao, T. S., Xu, J. B., \& Liang, Z. X. (2009). Journal of Power Sources, 187(1), 80-84.

[6] This study is supported by NSF awards No. 1507707 and No. 1560383, and NIH Build EXITO.

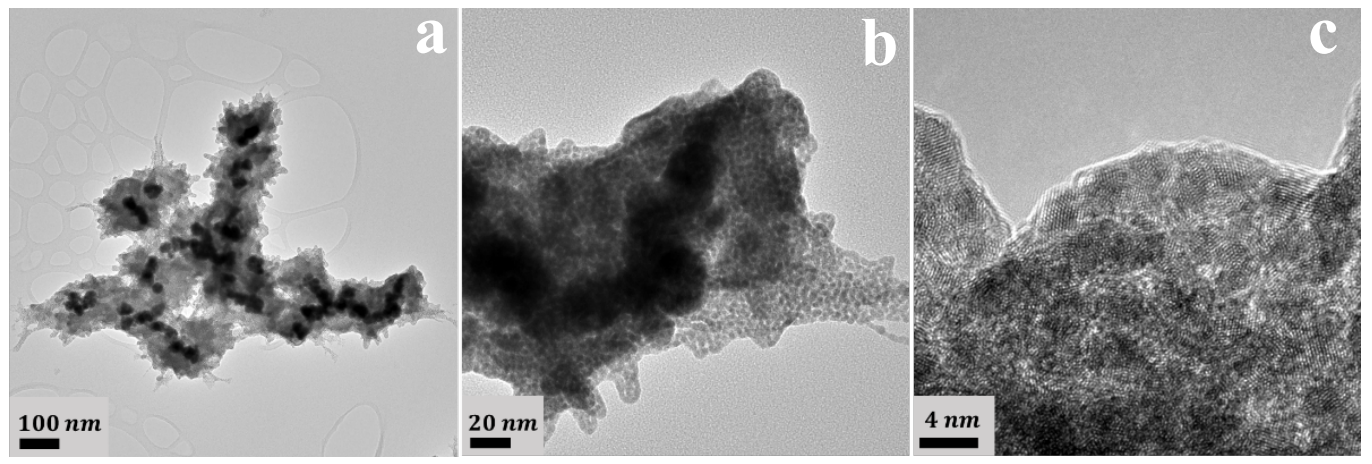

Figure 1 (a). Low magnification TEM images of Pd/Au NPs on graphene. (b) Higher magnification showing a core-shell structure. (c) NPs are crystalline. This sample had a 24-hour synthesis. A large cluster of Pd NPs surround Au core.
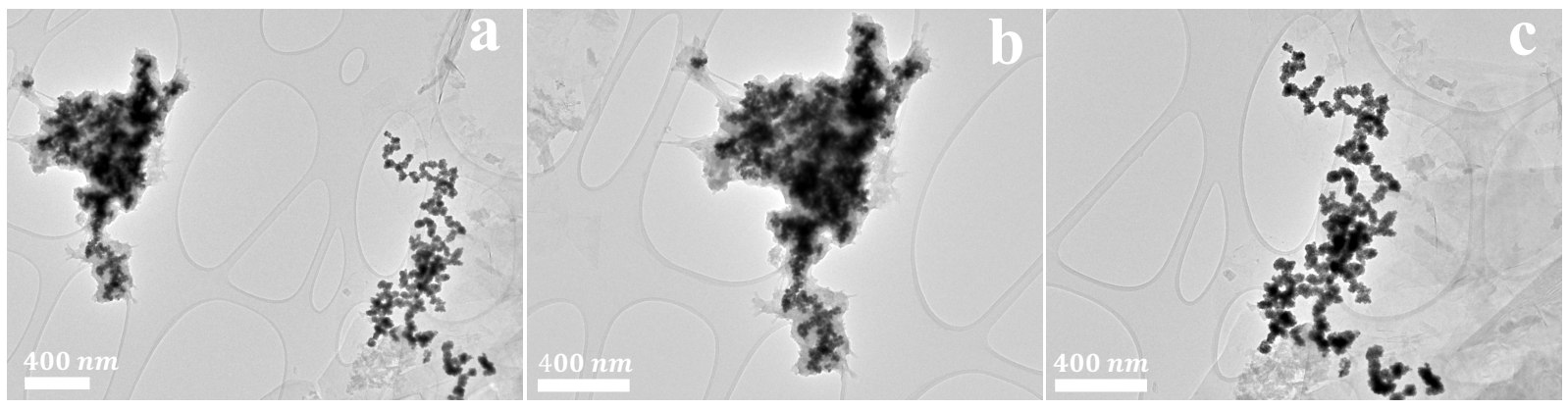

Figure 2 (a). Low magnification TEM images of Pd/Au NPs on graphene. While some NP clusters are fully formed (b), others have a thinner, not fully formed, Pd coating (c).
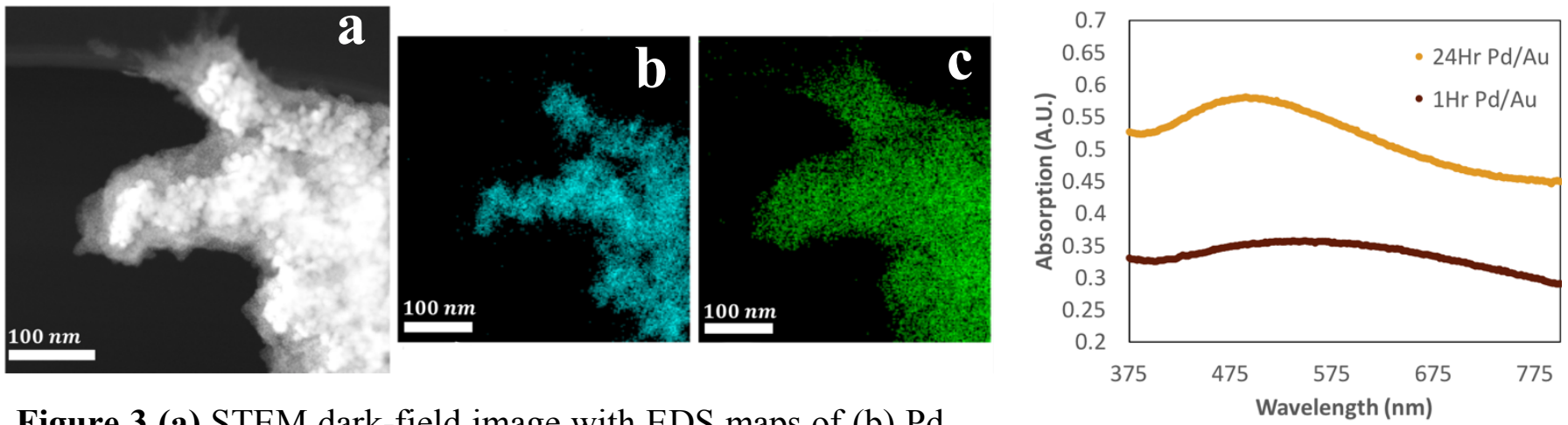

Figure 3 (a) STEM dark-field image with EDS maps of (b) Pd (green) and (c) $\mathrm{Au}$ (blue). This confirms $\mathrm{Pd}_{\text {shell }} \mathrm{Au}_{\text {core }}$ structure of the cluster.

Figure 4 UV-Vis analysis shows absorption is higher when NPs are given longer to react. 